\title{
Estudiantes de la Salud como Sujetos de Estudio en Investigación: Una Revisión Acerca de su Incorporación e Implicancias Éticas
}

\author{
Health Students as Study Subjects in Research: A Review \\ of their Incorporation and Ethical Implications
}

Nicolás Astete'; Alain Arias ${ }^{2,3}$ \& Ramón Fuentes ${ }^{2}$

\begin{abstract}
ASTETE, N.; ARIAS, A. \& FUENTES, R. Estudiantes de la salud como sujetos de estudio en investigación: una revisión acerca de su incorporación e implicancias éticas. Int. J. Morphol., 37(1):149-158, 2019.

RESUMEN: El propósito de este estudio fue conocer las implicancias involucradas sobre uso de estudiantes de la salud como sujetos de estudio en investigación. Se realizó una revisión de la literatura en las bases PUBMED, EMBASE, SCOPUS, EBSCOhost y SciELO, sin límites de años y con criterios de elegibilidad. Se encontraron un total 215 artículos, se realizó una selección de estos y se eliminaron estudios que se referían a otras poblaciones vulnerables o que evaluaban características psicológicas de los estudiantes sin una relación hacia la participación como sujetos de estudio. A excepción de un estudio cualitativo de entrevista, todos son estudios descriptivos de revisión narrativa, carta al editor o aplicación de encuestas, que expresan ciertas consideraciones que deben ser abordadas al utilizar estudiantes como sujetos de estudio. Es necesario realizar estudios en otras poblaciones de estudiantes y con una mayor rigurosidad en su metodología, y así poder evaluar el real beneficio educativo de su participación y sus percepciones sobre esta instancia. La sociedad científica hace un llamado a generar una guía que unifique los criterios que se deben considerar al seleccionar estudiantes como sujetos de estudio y así permitir que los investigadores, las instituciones de revisión ética y los editores de revistas científicas poder evaluar de mejor manera los estudios en estas poblaciones.
\end{abstract}

PALABRAS CLAVE: Estudiantes; Sujeto de investigación; Ética de la investigación; Poblaciones vulnerables.

\section{INTRODUCCIÓN}

Los numerosos incidentes que ocurrieron al experimentar con seres humanos el siglo pasado, exigieron una mayor rigurosidad en la supervisión de la investigación científica junto con la creación de pautas éticas para la protección de los seres humanos que participan como sujetos de estudio (SE) en investigación (Rice, 2008). El código de Nuremberg en 1947 (Nuremberg Military Tribunal, 1996) y la Declaración Universal de los Derechos Humanos (Oficina del Alto Comisionado para los Derechos Humanos, 2018), representan los primeros documentos redactados tras estos abusos que se refieren a los principales derechos de los participantes en investigación como: "voluntariedad y consentimiento informado", el "balanceado análisis de beneficios y riesgos de la investigación" y el "derecho a retirarse del estudio sin repercusiones". Estos documentos sirvieron de base para que la Asociación Médica Mundial redactara en 1964 la Declaración de Helsinki, la primera pauta con estándares éticos para la investigación biomédica con seres humanos (Dhai, 2014). En 1976 el Departamento de Salud, Educación y Bienestar de los Estados Unidos redacta el Informe de Belmont, documento que permitió regular la experimentación es este país y establecer los tres principios éticos fundamentales en el desarrollo de investigación que involucra seres humanos, el respeto por las personas, la beneficencia y la justicia. El primero alude a respetar la autonomía en la decisión a participar y a proteger aquellos indi-

\footnotetext{
${ }^{1}$ Programa de Magister en Odontología, Facultad de Odontología, Universidad de La Frontera, Temuco, Chile.

${ }^{2}$ Departamento de Odontología Integral Adultos, Centro de Investigación en Ciencias Odontológicas (CICO), Facultad de Odontología, Universidad de La Frontera, Temuco, Chile.

${ }^{3}$ Universidad Adventista de Chile, Chillán, Chile.
} 
viduos con una capacidad de decisión disminuida o deteriorada. Por otro lado el principio de beneficencia se refiere a no causar daño y proteger a los SE incrementando los beneficios y disminuyendo los posibles riesgos. Por último el de justicia impone el trato justo e igualitario de todos los potenciales participantes. No considerar alguno de estos involucra estar cometiendo una falta ética que sugiere modificar o redefinir la investigación al ser evaluada junto a una institución de revisión (IR) o comité ético científico (CEC) (Miracle, 2016).

A principios de la década de los 80’ y con la intención de "proporcionar principios éticos aceptados a nivel internacional junto con detallados comentarios sobre la manera de aplicar principios éticos universales", el Consejo de Organizaciones Internacionales de las Ciencias Médicas (CIOMS) en colaboración con la Organización Mundial de la Salud (OMS) redactan "Pautas éticas internacionales para la investigación relacionada con la salud con seres humanos", documento que incluye numerosas guías y reportes redactados desde la década del 50' y a través de su última actualización en el idioma español el año 2016, se convierte en una completa pauta ética internacional que guía el correcto uso de seres humanos en investigación en salud (van Delden \& van der Graaf, 2017).

En paralelo, otros países comenzaron a legislar la investigación en salud realizada en sus territorios. Dentro de las más de 1000 leyes gubernamentales redactadas en 130 países, Chile regula este actuar científico mediante tres: la Ley No 19.628, "Sobre la Protección de la Vida Humana", norma el correcto uso de los datos personales en investigación (Congreso Nacional, 1999). La Ley No 20.120 "Sobre la Investigación Científica en el Ser Humano, su Genoma y Prohíbe la Clonación Humana", protege la vida de los seres humanos en torno con la investigación científica biomédica (Congreso Nacional, 2006). Por último la Ley No 20.584 que "Regula los Derechos y Deberes que tienen las Personas en Relación con Acciones Vinculadas a su Atención en Salud", menciona la importancia de la voluntariedad, la implementación del consentimiento informado y el uso de poblaciones vulnerables (Congreso Nacional, 2012; Ahumada, 2012; Office for Human Research Protections, U.S. Department of Health and Human Services, 2018). Esta última ley en su artículo n²8 describe las implicancias de realizar investigación en grupos o poblaciones vulnerables (Valenzuela et al., 2015).

Los grupos de poblaciones vulnerables son clasificados por las pautas del CIOMS y definidos como individuos con una sustancial incapacidad para proteger sus propios intereses con la posibilidad de ser perjudicado física, psicológica o socialmente, dado por la disminución o au- sencia en su capacidad para tomar decisiones de manera voluntaria (Organización Panamericana de la Salud \& Consejo de Organizaciones Internacionales de las Ciencias Médica, 2016). Dentro de la clasificación que ofrece estas pautas, una relación jerárquica de por medio representa una categoría de individuos bajo una condición de subordinación denominada relación de dependencia, que influye en la toma de decisión libre y voluntaria de participar de una investigación (Organización Panamericana de la Salud \& Consejo de Organizaciones Internacionales de las Ciencias Médica). Ejemplos son el personal subordinado de hospitales y laboratorios, trabajadores de pauta $\mathrm{n}^{\circ} 9$, en centros de investigación o de las fuerzas armadas o la policía y los estudiantes en el ámbito académico (Organización Panamericana de la Salud \& Consejo de Organizaciones Internacionales de las Ciencias Médica).

Parte del continuo crecimiento de la investigación médica educacional es realizar investigación con estudiantes, donde su participación como SE puede convertirse en la primera instancia de acercamiento a la ciencia, y por ende, la oportunidad para la adquisición de nuevo conocimiento (Aycock \& Currie, 2013). Dentro de la investigación realizada en universidades es reconocida la fácil accesibilidad de los estudiante para aceptar participar como SE, pero su falta de vinculación al área, trae consigo el desconocimiento de los posibles riesgos y beneficios involucrados en su participación, viéndose comprometida su autonomía para consentir libremente debido a la posición de autoridad del investigador responsable, creyendo que aceptar o negar la participación puede generar mejores o peores oportunidades (Christakis, 1985; Forester \& McWhorter, 2005). Es responsabilidad del investigador no influir en esta decisión ni exponer a un mayor riesgo a los voluntarios, esto los obliga a conocer las estrategias de protección para su incorporación a través de la evidencia científica, la reglamentación local y los protocolos éticos internacionales que rigen la investigación con seres humanos (Sullivan, 2011; Sample et al., 2013; World Medical Association, 2013).

La siguiente revisión tiene por objetivo conocer las temáticas involucradas al incorporar estudiantes del área de la salud como SE en investigación y la protección que se les debe otorgar.

\section{MATERIAL Y MÉTODO}

Bases de datos. Se realizó una revisión de la literatura con búsqueda sistematizada sin limites de años, de acuerdo al protocolo PRISMA (Moher et al., 2009). Las bases de datos empleadas incluyeron PUBMED, EMBASE, 
SCOPUS, EBSCOhost y SciELO. Los términos de búsqueda utilizados fueron "students", "dental students", "medical students", "research subject", "subject of research", "educational research", "dental research", "medical research" y "biomedical research". Los términos fueron utilizados individualmente y en combinación a través de operadores boléanos. La búsqueda fue realizada entre enero y marzo de 2018.

Criterios de selección. El propósito de la revisión es reconocer las distintas dimensiones involucradas al incorporar estudiantes de las ciencias de la salud como SE en investigaciones, reconociendo como la dimensión principal de investigación: "Participación de estudiantes como SE". Previo a realizar esta revisión se observó, mediante una búsqueda simple en la literatura, la importancia de respetar los principios éticos al plantear una investigación en estudiantes, como también, que esta participación representa un método de enseñanza con variados beneficios para los participantes e investigadores. Abordar estas perspectivas se consideró un criterio de inclusión, sin embargo, no se excluyeron artículos que aportaban en otro aspecto antes no reconocido.

Los criterios de inclusión fueron: (1) artículos sobre a las implicancias éticas, educativas y otras, al investigar en estudiantes de las ciencias de la salud, (2) artículos en inglés o español, (3) estudios cualitativos (4), estudios des-

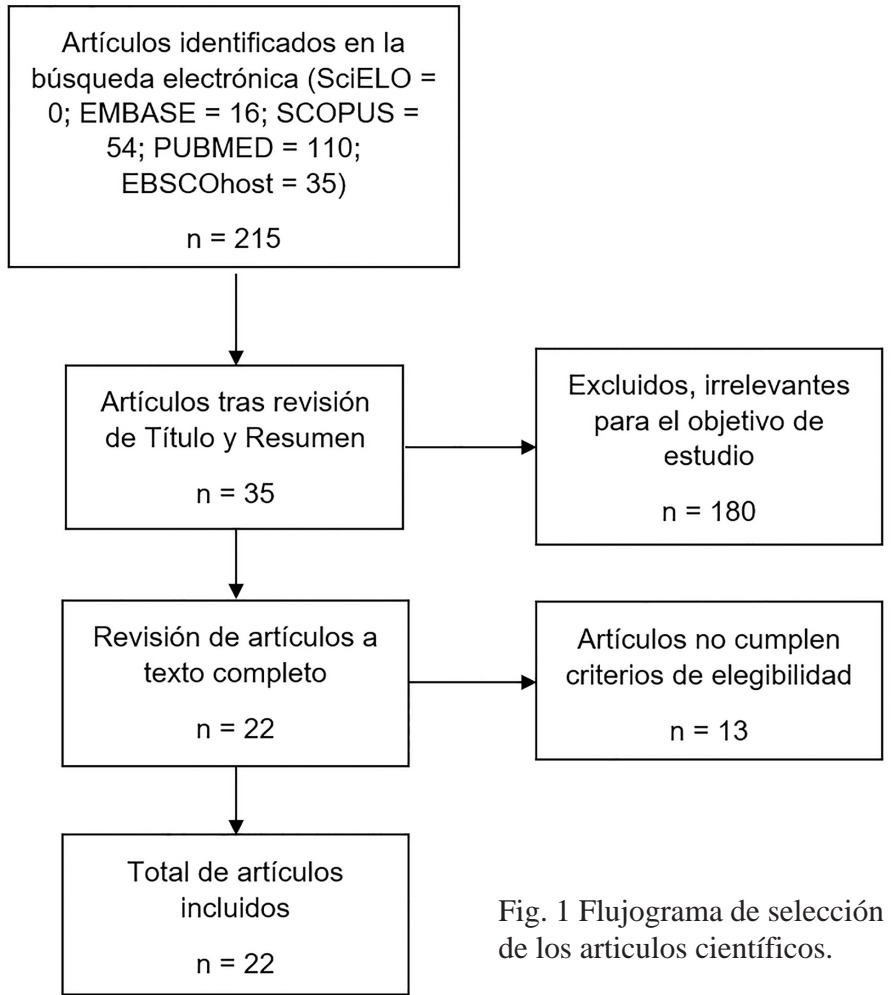

criptivos tipo revisión de la literatura, guías y protocolos, estudios de creación, validación o aplicación de encuestas y cartas al editor. Se excluyeron artículos centrados en estudiar otras poblaciones vulnerables, artículos enfocados en los beneficios de la investigación científica para el estudiante sin mencionar su rol como SE, características psicológicas de los estudiantes sin relación con la participación como SE, evaluación de electivos de metodología científica en estudiantes. Adicionalmente se revisó la lista de referencias de los estudios seleccionados.

\section{RESULTADOS}

Se encontraron 215 artículos en las cinco bases de datos revisadas, que luego de la revisión por título y resumen, 35 de ellos fueron seleccionados para una revisión de texto completo (Fig.1). Tras aplicar los criterios de inclusión, 22 artículos aportaban información en relación a las consideración que se deben conocer al utilizar estudiantes como SE.

Descripción de los estudios. En relación a los artículos excluidos estos en su mayoría se enfocaban hacia el estudio de variables psicológicas de los estudiantes hacia la investigación y no hacia la participación como SE, y otros en representar a otras poblaciones vulnerables como mujeres, embarazadas, pacientes, tercera edad y trabajadores.

De los artículos seleccionado trece son revisiones narrativas, tres cartas al editor, tres aplicaciones de encuesta, un estudio de creación y validación de una encuesta y un estudio cualitativo.

Tras evaluar la evidencia, tres fueron los temas más representados por los autores. Todos los artículos vinculan algún ámbito de la ética científica al involucrar estudiantes como SE en investigación. De los dieciséis autores que se refieren respecto a las presiones indebidas, voluntariedad o coerción (Shannon, 1979; Christakis; Diamond \& Reidpathd, 1992; Dalziel, 1996; Clark \& McCann, 2005; Forester \& McWhorter; Ferguson et al., 2006; Ridley, 2009; Voo, 2009; Bradbury-Jones \& Alcock, 2010; Sykes \& Dullabh, 2012; Leentjens \& Levenson, 2013a; Aycock \& Currie; Sarpel et al., 2013; Vaidya et al., 2016; Boileu et al., 2017), once son revisiones narrativas o publicaciones en ética científica (Shannon; Christakis; Dalziel; Clark \& McCann; Ferguson et al.; Ridley; Bradbury-Jones \& Alcock; Sykes \& Dullabh; Leentjens \& Levenson, 2013a; Aycock \& Currie; Boileu et al., 2017), tres 
son aplicaciones de encuestas a estudiantes de las ciencias de la salud (Diamond \& Reidpathd; Forester \& McWhorter; Vaidya et al.), uno es una carta al editor (Voo) y uno es un estudio cualitativo (Sarpel et al.).

Diez artículos se refieren a temas de confidencialidad y anonimato para disminuir los riesgo de la participación de los estudiantes (Shannon; Christakis; Diamond \& Reidpath; Forester \& McWhorter; Clark \& McCan; Ferguson et al.; Ridley; Bradbury-Jones \& Alcock; Aycock \& Currie; Boileau et al., 2017), donde ocho son revisiones narrativas (Shannon; Christakis; Clark \& McCann; Ferguson et al.; Ridley; Bradbury-Jones \& Alcock; Aycock \& Currie; Boileau et al.) y dos aplicaciones de encuestas (Diamond \& Reidpath; Forester \& McWhorter).

Ocho artículos se refieren a ser justos con los estudiantes en su participación (Shannon; Christakis; Clark \& McCann; Ferguson et al.; Ridley; Sykes \& Dullabh; Sarpel et al.; Boileau et al.), siete son revisiones narrativas (Shannon; Christakis; Clark \& McCann; Ferguson et al.; Ridley; Sykes \& Dullabh; Boileau et al.) y uno es un estudio cualitativo (Sarpel et al.).

El segundo tema es sobre los "beneficios que trae su participación como SE donde ocho de los veintidos autores se refieren a él (Shannon; Dalziel; Forester \& McWhorter; Aycock \& Currie; Leentjens \& Levenson, 2013a; Roberts \& Allen, 2013; Sarpel et al.; Walsh, 2013). Cinco articulos fueron revisiones narrativas o publicaciones en ética científica (Shannon; Dalziel; Aycock \& Currie; Leentjens \& Levenson, 2013a; Roberts \& Allen), una aplicación de encuesta (Forester \& McWhorter), un estudio cualitativo (Sarpel et al.) y una carta al editor (Walsh).

El último tema abarcado es sobre la "responsabilidad de las instituciones de revisión o comités éticos" con ocho de los veintidós articulos (Henry \& Wright, 2001; Forester \& McWhorter; Ferguson et al.; Scheppler \& Kolar, 2008; Sarpel et al.; Leentjens \& Levenson, 2013a,b; Boileau et al.), donde cinco son revisiones narrativas (Henry \& Wright; Ferguson et al.; Scheppler \& Kolar; Leentjens \& Levenson, 2013a; Boileau et al.), una encuesta (Forester \& McWhorter), un estudio cualitativo (Sarpel et al.), y una carta al editor (Leentjens \& Levenson, 2013b).

\section{DISCUSIÓN}

Implicancias éticas de la participación de estudiantes como sujetos de estudio. Al considerar a los estudiantes como SE surgen consideraciones éticas particulares que abor- daremos a través de los principios éticos fundamentales en el desarrollo de investigación que involucra seres humanos, el respeto por las personas, la beneficencia y la justicia. Estos conceptos deben ser considerados por los investigadores en el transcurso de todo un estudio (Rice; Boileau et al.).

Principio de respeto por las personas. Al no considerar el principio de respeto por los estudiantes, involucra violar también otros dos criterios éticos, la autonomía y la vulnerabilidad de los participantes (Miracle). Estos conceptos deben ser abarcados durante el proceso de consentimiento informado, instancia donde se entrega toda la información necesaria al estudiante para que pueda generar una completa e informada decisión, además de asegurar que serán respetadas sus decisiones durante todo el transcurso de la investigación sin manipulación o influencia, enfatizando la incorporación completamente voluntaria al estudio (Aycock \& Currie). Presiones indebidas hacia los participantes deben ser evitadas, como lo es una relación jerárquica o autoritaria con el investigador responsable y el pago indebido o sanción por la participación, situaciones que pueden generar coerción o influencias en la decisión de aceptar o rechazar participar de un estudio y la percepción de que su decisión pueda generar mejores calificaciones, créditos, recomendaciones, reconocimiento o incluso sanciones graves y rechazo social (Shannon; Christakis; Dalziel; Clark \& McCann; Forester \& McWhorter; Ferguson et al.; Ridley; Sykes \& Dullabh; Sarpel et al.; Aycock \& Currie; Vaidya et al.; Organización Panamericana de la Salud \& Consejo de Organizaciones Internacionales de las Ciencias Médica; Boileu et al., 2017). Estas situaciones coercitivas han sido observadas en otras instancias, así lo reporta Diamond \& Reidpathd donde observaron cómo ciertos incentivos y sanciones eran impuestas a los estudiantes de psicología para presionar su decisión a participar. Incluso se observó que algunas instituciones incorporaban esta acción de forma obligatoria al ingresar al plan de estudio, generando ocasiones donde los estudiantes nunca se enteraron de este mandato hasta tener avanzado su plan de estudios (Diamond \& Reidpathd).

Esta cercanía con el investigador se denomina relación de dependencia y genera que estos participantes sean clasificados como una población vulnerable en investigación, categorizada así por las pautas del CIOMS (Organización Panamericana de la Salud \& Consejo de Organizaciones Internacionales de las Ciencias Médica). Es necesario reconocer la presencia de estas característica al plantear una investigación y adoptar las medidas suficientes para disminuir los potenciales riesgo en su participación como SE. Christakis explica como la relación de dependencia genera una coerción que altera el proceso de consentimiento informado, viéndose reprimida la autonomía del estudiante en 
el proceso. Comenta tener cuidado al ofrecer incentivos monetarios ya que la motivación del estudiante debe estar en la oportunidad de aprender y contribuir y no en la recompensa financiera (Christakis). Por otro lado propone, en base a lo descrito por Shannon, evitar una invitación individual y realizar en cambio una invitación de manera general, a un gran número de potenciales participantes, como estrategia para evitar una presión indebida (Shannon; Christakis; Aycock \& Currie). Dalziel también observa como este proceso de consentimiento informado se ve alterado y comenta como la cercana relación entre la investigación y la academia ha provocado que los estudiantes formen parte de investigaciones sin siquiera saberlo, ya sea dentro de un test diagnostico o examen en clases. Propone reconocer las relaciones de dependencia y reforzar el proceso de consentimiento informado, haciendo que este sea más claro, sencillo, y expresado con un lenguaje apropiado que facilite la mejor comprensión por los participantes tanto de los objetivos del experimento como de sus derechos y expectativas de su participación (Dalziel). Defiende además el valor educativo de esta experiencia argumentando que esta no es más coercitiva que otros requisitos de los cursos o asignaturas, como lo es un examen o test (Dalziel).

Clark \& McCann mencionan otra medida para evitar la coerción. Luego de recibir las enmiendas propuestas por una institución de revisión a la cual enviaron a evaluación ética un proyecto de investigación, toman la decisión de capacitar a un profesor, que no formara parte del claustro de investigadores, y que representara un tipo de intermediario entre los participantes y científicos, este se encargaría de realizar la fase de reclutamiento y administración de los cuestionarios, esto dentro del horarios de clases de los alumnos, con la salvedad de no informar a los miembros de la investigación detalles como quiénes aceptaron o denegaron participar u otros datos que pudieran identificar a los participantes ante los investigadores (Clark \& McCann). No se refieren al uso de los horarios de clases de los estudiantes, lo que puede representar un potencial riesgo en su participación. Ferguson et al. y Ridley, en el área de la enfermería, también reconocen esta estrategia para evitar la coerción en investigación con sus estudiantes, enfatizando que la etapa de reclutamiento, proceso de consentimiento informado e incluido el análisis de los datos, debe ser llevada a cabo por otro investigador, co-investigador o asistente, que no presente una relación de dependencia con los potenciales participantes y que además se debe dejar explícitamente escrito que esta decisión fue voluntaria y sin presión (Ferguson et al.; Ridley). Ridley y Aycock \& Currie también están de acuerdo en evitar la coerción a través de la incorporación de un tercer actor que no posea una relación que comprometa la decisión de los estudiantes, el que estará encargado de realizar la invitación general a los estudiantes a través de anuncios y publicidad, evitando así reclutar de manera individual. Recomiendan además otorgar mínimo 24 horas para tomar la decisión de participar o rechazar, también de incluir de manera textual en el documento de consentimiento el carácter voluntario de la participación y que el rechazo no trae repercusiones para el estudiante, y por último que se debe identificar a los responsable del estudio como investigador y no como profesor (Aycock \& Currie). Bradbury-Jones \& Alcock proponen una lista de tópicos para investigadores en enfermería, la cual pueden consultar con el fin de reconocer si los estudiantes entiende tres dimensiones sobre participar como SE: "la contribución de la investigación, relaciones en la investigación y el impacto de la investigación". Es en el punto sobre las "relaciones en la investigación" donde el estudiante debe diferenciar muy bien cuál es el rol que cumple el profesorinvestigador responsable, sobre todo en investigación cualitativa donde se establecen entrevistas presenciales (Bradbury-Jones \& Alcock). Recomiendan incluir entre paréntesis, luego da cada una de sus firmas en los documentos, la etiqueta de "investigador en enfermería" (o área del conocimiento), y con esto reforzar el rol de investigador que está desarrollando sobre su rol de académico (Bradbury-Jones \& Alcock; Leentjens \& Levenson, 2013a).

El uso de incentivos por la participación deben ser cuidadosamente evaluados evitando ofrecer montos excesivos y asegurando una alternativa extra para obtener el mismo incentivo en aquellos que rechazan participar (Aycock \& Currie). Otros autores recomiendan evitarlos, siendo aceptable el reembolso a los participantes por gastos de locomoción y estacionamiento como también el proveer alimentos que forman parte la rutina diaria (Ferguson et al.; Ridley; Sykes \& Dullabh). Si se opta por incorporar un incentivo en la participación, es recomendable utilizar pagos en dinero antes que otorgar crédito extra o mejor calificación, esto permite que los participantes perciban el beneficio monetario de manera similar, al contrario de lo que un crédito extra o mejor calificación puede generar en las necesidades académicas de los alumnos (Voo; Sykes \& Dullabh). El uso de algún tipo de incentivo debe ser analizado en conjunto a una institución de revisión ético científico (Sykes \& Dullabh). Pero contrario a estas ideas, Sykes \& Dullabh, Leentjens \& Levenson (2013a) y Boileau et al. concluyen que no deben ofrecerse pagos indebidos de tipo monetarios o académicos, tampoco se deben generar consecuencias para el estudiante por negarse a participar, y este rechazo no debe ser compensado con alternativas para lograr el mismo incentivo, ya que esta estrategia puede vulnerar la privacidad, generan desigualdad en los tiempos invertidos, esfuerzo involucrado y beneficios educativos entre los estudiantes que aceptan y rechazan participar. 
Boileau et al. publica en una revista médica las 12 cuestiones éticas que deben ser consideradas al involucrar estudiantes como SE. Aquí recomienda que la justificación de solicitar el consentimiento informado se ha establecido antes de la aplicación del estudio, junto a un comité de revisión, asegurando que la potencial exposición de información identificable de los sujetos esta resguardada. También agrega el uso de otro individuo calificado y completamente independiente de poseer alguna relación con el estudiante para el reclutamiento y consentimiento informado y recomienda un sistema online de reclutamiento, SONA-system que otorga mayor privacidad y resuelve el tema de la presión indebida (Leentjens \& Levenson, 2013a; Boileau et al.). Es importante no influir indebidamente en la decisión de los participantes y asegurar su confidencialidad para permitir una mayor veracidad de los datos entregados y evitar el sesgo de respuesta por los participantes (Boileau et al.).

Los estudios observacionales de Forester \& McWhorter mediante una encuesta a estudiantes de medicina, y lo reportado por Sarpel et al. en un estudio cualitativo a través de un grupo focal, no observan la presencia de coerción en la decisión de los estudiantes por la participación en investigación como SE. Estos resultados se contrastan a lo documentado por Vaidya et al. quien también a través de una encuesta, esta vez en una población india de estudiantes de medicina, reveló proporciones más altas de esta influencia. Esto evidencia como difieren las percepciones de los estudiantes en diferentes poblaciones y la limitada evidencia empírica que aún existe en esta temática. Sumado a esto Leentjens \& Levenson (2013a) mencionan que debido a la falta de acuerdo en la literatura sobre qué criterios se deben seguir al plantear una investigación con estudiantes como SE, hacen un llamado a la sociedad científica por unificar internacionalmente estos estándares e incorporarlos tanto en las instituciones de revisión en ética como en las editoriales de revistas científicas y así guiar correctamente las investigaciones que involucran la participación de estudiantes como SE.

\section{Principio de Beneficencia}

Es importante asegurar el bienestar de los participantes en una investigación. Dentro de las consideraciones éticas que se deben tener en cuenta para respetar el principio de beneficencia están no hacer daño y disminuir los riesgo junto con a incrementar los potenciales beneficios de la participación (Miracle). Es necesario respetar la privacidad de los estudiantes asegurando la confidencialidad de toda la información presente en el estudio, en especial en la recolección, análisis y uso de los datos, junto con asegurar el anonimato de los participantes (Christakis; Ridley; Boileau et al.), Shannon agrega evitar intervenciones físicas y quirúrgicas de mayor complejidad al incluir estudiantes como SE.
Mantener la confidencialidad de los datos, toma esencial importancia cuando nos referimos a investigación educacional, donde en ocasiones se analiza información privada o sensible de los estudiantes, que sumado a que pertenecen a una acotada y cerrada comunidad médica universitaria, cierta información demográfica y el prolongado contacto entre los investigadores y los alumnos, expone al estudiante a ser identificado en su comunidad (Christaki, 1985; Clark \& McCann; Forester \& McWhorter; Bradbury-Jones \& Alcock; Organización Panamericana de la Salud \& Consejo de Organizaciones Internacionales de las Ciencias Médicas). Al preguntar a 524 estudiantes de medicina en una universidad de Kansas, Estados Unidos, respondieron no estar preocupados por la confidencialidad de la información cuando se aseguraba que su participación era voluntaria y anónima (89\%) (Forester \& McWhorter). Para asegurar esto, es necesario analizar ciertas características de la población objetivo. Analizar un gran número de estudiantes para asegurar una mejor confidencialidad de los datos es esencial (Ferguson et al.). Clark \& McCann muestran como se mantiene la confidencialidad en un cuestionario administrado a estudiantes de segundo y tercer años de medicina de tres diferentes campus, realizando únicamente tres preguntas demográficas sobre género, avance curricular y rango de edad, evitando indagar el campus al que pertenecían y mezclando las respuestas de estos tres sectores. Justifica esta acción con el fin evitar la identificación de la poca cantidad de alumnos varones distribuidos en los departamentos universitarios y evitar hacer comparaciones entre estos tres sectores (Clark \& McCann). Además, para asegurar no conocer la identidad de quienes no decidieron participar, todos los estudiantes debieron depositar un sobre con la respuesta a esta invitación en un buzón, donde el cuestionario en blanco significó el rechazo (Clark \& McCann).

Junto con evitar una presión indebida en las respuestas de los estudiantes, la recolección y análisis de los datos debe ser llevaba a cabo por un individuo sin una relación dependiente con los participantes, esto fortalece el anonimato del estudio y evita que el profesor-investigador directo de los estudiantes tenga alguna relación con la información o que influya en las respuestas del estudiante, alumno que puede pensar que una respuesta afirmativa o negativa influye en su relación con el académico, poniendo en duda la veracidad de los datos obtenidos (Diamond \& Reidpath; Ferguson et al.). Esto no excluye que si la información obtenida en el estudio indica que el participante está en peligro, esta deba ser avisada confidencialmente a este (Bradbury-Jones \& Alcock).

Sin embargo, es fundamental eliminar todo dato identificador de la información obtenida y codificarla para así permitir el completo anonimato (Aycock \& Currie; 
Boileau et al.). Boileau et al. comenta, que es fundamental que toda la información de un estudio sea resguarda de manera segura de algún acceso sin autorización, para esto es necesario decidir tanto el lugar físico donde se protegerá, quienes tendrán acceso a ella y las técnicas que aseguran su identificación, acceso y perdida, como la codificación, el uso de contraseñas computacionales y antivirus. Especial cuidado debe otorgarse a investigaciones de encuestas vía email, asegurando encriptar los datos o elegir software especializados. En encuestas online, es necesario revisar losreglamentos de seguridad de las páginas web respecto a la codificación de los datos y firewalls (Boileau et al.).

Principio de Justicia. El trato justo e igualitario para todos los participantes es la principal tarea al respetar el principio de justicia, que debe generar confianza absoluta entre el estudiante e investigador/ profesional de la salud/ académico (Miracle). Considerar seleccionar estudiantes por lo factible que es su participación es inadecuado y se han de elegir basados en los objetivos de investigación, no por conveniencia (Clark \& McCann; Ridley).

Respetar este principio involucra realizar un adecuado plan de muestreo, establecer correctamente los criterios de elegibilidad, realizar la aleatorización de la intervención, asegurar que el rechazo o retiro del estudio no traerá sanciones ni represalias y considerar el tiempo involucrado en la participación (Ridley; Miracle). Estos últimos dos puntos toman mayor importancia al plantear una investigación con estudiantes como SE, en quienes puede surgir una sensación de frustración al considerar que su participación pueda generar el gasto innecesario de su tiempo o la pérdida de oportunidades, siendo imperante considerar el tiempo que el estudiantes dedicará al estudio, sobre todo el utilizado durante la fase de reclutamiento y recolección de los datos (Sykes \& Dullabh). Este lapso de participación no debe invadir la vida diaria del estudiante ni generarle conflictos, respetando principalmente su horario estudiantil y actividades universitarias obligatorias (Christakis; Boileau et al.). Sin embargo Christakis agrega que otorgar una protección extra a los estudiantes solamente por pertenecer a un grupo en particular, puede ser una medida sobre protectora y paternalista. Shannon considera que no se debe sobrepasar una semana por semestre de participación para extensos estudios.

Por otro lado, Clark \& McCann dieron solución a las enmiendas realizadas por una institución de revisión respecto al tiempo que empleaba responder una encuesta que se administraría a una población de estudiantes de enfermería. Este cuestionario se impartiría a principio de semestre académico dentro de las clases de las asignaturas de educación y promoción de salud (Clark \& McCann). Boileau et al. recomienda utilizar los espacios libres entre las actividades educativas obligatorias como también los tiempos libres tras exámenes, sin olvidar evaluar siempre estas medidas junto a un comité ético científico.

En cuanto al rechazo por participar o retiro de la investigación, se debe asegurar que la decisión de los estudiantes sea completamente confidencial y sin sanción, dejando esto por escrito en el documento de consentimiento informado como también reconociendo el entendimiento por parte del participantes de dicha sentencia (Ferguson et al.; Sykes \& Dullabh). Por último, los investigadores deben tener particular cuidado en asegurar que no existe discriminación en sus estudios ya sea a través del lenguaje expresado como en los instrumentos de medición utilizados o el reporte de los resultados (Sykes \& Dullabh).

Seguir estas medidas es fundamental para asegurar que los datos obtenidos en el estudio son verídicos, despojados de sesgo de respuesta, y que la experiencia de participar como SE para el estudiante es satisfactoria (Sarpel et al.).

Ser sujetos de estudio: beneficio para la investigación y para el estudiante. Ya establecidas las primeras Instituciones de Revisión o los primeros Comités Éticos Científicos en los Estados Unidos, Thomas Shannon en 1979 expone las características que hacen factible la investigación con estudiantes del área de la salud como SE, que se continúan observando en la investigación moderna. En cuanto a los beneficios otorgados a la realización de la investigación, estos estudiantes son parte de la población adulta capaz de tomar sus propias decisiones, son de fácil localización en las instituciones donde estudian, disponen en su mayoría de buen estado de salud, poseen un desarrollo intelectual que permite llevar a cabo un mejor proceso deconsentimiento informado y pueden otorgar un detallado reporte de los efectos de las terapias de estudio (Shannon). En cuanto a los beneficios para el estudiante, su participación les permite conocer cómo se realiza la fase experimental, el método científico y beneficiarse intelectualmente de esta incorporación (Shannon). Dalziel, en una revista australiana de psicología, afirma que la participación activa en el aprendizaje, como es la incorporación como SE en investigación, permite al estudiante estar más atento, retener mejor la información y disfrutar de lo enseñado, a diferencia de lo que es la participación pasiva o lecturas en el aprendizaje. Así también, da algunos ejemplos sobre este beneficio intelectual, del cual pueden obtener cierto conocimiento de los procedimientos y condiciones experimentales reales, sirviendo de base para que los estudiantes puedan diseñar mejor su propia investigación en los últimos años de pregrado y aquellos que participan en más de un proyecto de investigación, pueden reflexionar sobre las diferencias en la metodología de investi- 
gación entre su participación o cómo algunos proyectos son inevitablemente más rigurosos que otros (Dalziel). De esta forma la participación como SE en investigación puede convertirse en una nueva técnica de enseñanza (Dalziel).

Aycock \& Currie en una revisión bibliográfica de educación en enfermería, también reportan los beneficios tanto para la investigación como para el potencial participante, reconociendo también la accesibilidad de los estudiantes, la cantidad de estudiantes presentes para constituir muestras significativas, ser generalmente pacientes sanos sin enfermedades crónicas graves, que presentan una educación que les permite comprender de mejor manera las características del estudio y agrega la variedad en etnia y edad que los convierte en una población atractiva para desarrollar investigación. Por otro lado el estudiante puede beneficiarse de la investigación a través de la experiencia educativa vivida y en ocasiones, la posibilidad de acceder a algún examen de salud, como un hemograma o un examen de colesterol, sin costo alguno (Aycock \& Currie).

Christakis en 1985 reporta como la investigación educacional y el uso de estudiantes como sujetos de estudio en medicina a sido necesario para demostrar al estudiante la doble importancia que su participación aportar a la ciencia, uno a través de mejorar la salud y el otro al beneficiar la educación universitaria. Sin embargo este es un rol sin beneficios directos para el estudiante y solamente algo intrínseco del método científico (World Medical Association). En el ámbito de la educación psicológica, la evidencia empírica que respalda los beneficios directos de la participación es escasa, así lo reporta Leentjens \& Levenson (2013a) quienes muestran que el único estudio que compara la técnica activa de aprendizaje en psicología, no resultó ser más beneficiosa que la técnica pasiva de lecturas en clases (Eliott et al., 2010). Justificarlo como un beneficio para el estudiante es inapropiado y enfocado solamente en conseguir una muestra de participantes con el menor gasto de tiempo y recursos (Leentjens \& Levenson, 2013a). Sumado a esta idea, Roberts \& Allen indican que los ensayos clínicos en psicología que respaldan esta información raramente se estudian o no se evalúan adecuadamente, siendo muy limitados, por lo que deciden fabricar y validar una herramienta psicométrica para medir esta dimensión (Roberts \& Allen).

En educación médica, Forester \& McWhorter a través de una encuesta a estudiantes de pregrado de medicina (58,5\% de respuesta), evaluó sus percepciones respecto a la investigación educacional, la participación como SE y su relación con los investigadores responsables, donde el $93 \%$ de los estudiantes consideró importante la investigación médica educacional para contribuir en su mejoría, pero con un contradictorio $40 \%$ que consideró estar de acuerdo en participar en investigaciones como SE (Forester \& McWhorter). Ellos sugieren continuar el estudio en otras poblaciones de estudiantes, adecuando los cuestionarios o generando nuevos instrumentos de medición (Forester \& McWhorter).

Un estudio cualitativo en estudiantes de medicina de Sarpel et al., analizó la respuesta de los estudiantes respecto a su percepción como participantes en una encuesta previamente realizada, donde los estudiantes respondieron que sentir que estaban aportando a la investigación educacional a pesar de no ser directamente beneficios de la investigación, consideraron importante esta participación para sus futuras interacciones con el procesos científico y sintieron que representaba un conocimiento que necesitaban obtener (Sarpel et al.). Sin embargo la muestra de este estudio podría no ser representativa de la población objetivo de estudiantes de medicina seleccionada, principalmente por falta de representatividad en el focus group (Walsh).

Por último, para reforzar de manera educativa la participación del estudiante como SE, Nimmer \& Handelsman (1992) proponen entregar a los participantes un acotado documento donde puedan consultar los temas más relevantes de la investigación junto con una o dos referencias bibliográficas lo que enriquecería la experiencia de aprendizaje (Nimmer \& Handelsman).

Tanto la investigación educacional de alguna ciencia, como el estudiante al ser SE pueden beneficiarse del método científico bien realizado. Sin embargo los estudios que respaldan estos beneficios son escasos, basados principalmente en las percepciones de los estudiantes o sin representar una medición adecuada del tema. Basados en los estudios previos es necesario generar nuevas investigaciones de mejor representatividad en las poblaciones y generar nuevos métodos de medición de esta dimensión comparando los resultados.

Evaluación por una institución de revisión científica. La evaluación de un proyecto por una institución de revisión o comité ético científico es esencial y beneficioso, ya que permite asegurar la protección de los participantes, principalmente en temas sobre sus potenciales riesgos, confidencialidad y justicia (Ferguson et al.).

Ya sea al plantear un programa educativo para mejorar el método de enseñanza de una asignatura o realizar un proyecto de investigación que termine en la publicación, si estos involucran estudiantes como SE, debe ser enviado a revisión por un comité ético científico (Henry \& Wright). La evaluación exhaustiva del proyecto será solicitada cuando este estudio indique que generará conocimientos que se expondrán a la sociedad, ya sea a través de la publicación en revis- 
tas científicas o cualquier otro medio de difusión que exponga la privacidad de los participantes, exigiendo a los investigadores la aplicación de un proceso de consentimiento informado (Henry \& Wright; Scheppler \& Kolar). Pero las constantes discrepancias encontradas entre las evaluaciones realizadas por distintas instituciones al enviar un mismo proyecto tienen a los investigadores menos interesados en investigación educativa (Sarpel et al.; Boileau et al.). Forester \& McWhorter afirman que una revisión exhaustiva es innecesaria e inapropiada al observar que los alumnos de medicina encuestados en su estudio no se sintieron presionados a participar ni sintieron preocupación acerca de su confidencialidad si se aseguraba que su participación era completamente voluntaria y anónima (Forester \& McWhorter). La unificación de criterios y la creación de un estándar internacional al evaluar estas investigaciones es fundamental y debe ser incorporada tanto por las instituciones de revisión como las editoriales de las revistas científicas (Leentjens \& Levenson, 2013a,b).

Por último, la incorporación de uno o dos expertos en estudios con poblaciones vulnerables por parte de las instituciones de revisión es fundamental al evaluar una investigación que involucra estudiantes y es una medida que debe ser aplicada por las instituciones de revisión (Boileau et al.).

\section{CONCLUSIÓN}

La participación de los estudiantes como sujetos de estudio en investigación puede convertirse en una instancia académicamente enriquecedora, pero es escasa la evidencia que reporta los verdaderos beneficios estudiantiles de su participación.

El uso de estudiantes como sujetos de estudio a incrementado con el paso de los años, exigiendo a los investigadores conocer las implicancias éticas involucradas en su participación. Reconocer las medidas para su protección es fundamental y los científicos hacen un llamado a unificar internacionalmente estos criterios para dar solución a las frecuentes discrepancias observadas al solicitar la evaluación de este tipo de proyectos por diferentes instituciones de revisión, lo que beneficiará también a los investigadores y editores de revistas científicas.

ASTETE, N.; ARIAS, A. \& FUENTES, R. Health students as study subjects in research: A review of their incorporation and ethical implications. Int. J. Morphol., 37(1):149-158, 2019.

SUMMARY: The aim of this study was to know the implications of including health students as subjects of research study. A review of the literature was carried out in the databases
PUBMED, EMBASE, SCOPUS, EBSCOhost and SciELO, without limits of years and with eligibility criteria. A total of 215 articles was found, a selection was made of these, and studies were eliminated that referred to other vulnerable populations, or that evaluated psychological characteristics of the students, with no relation to their participation as subjects of the study. With the exception of a qualitative interview study, all are descriptive studies of narrative reviews, letters to the editor, or surveys, which express certain considerations that must be addressed when using students as study subjects. It is necessary to carry out studies in other student populations, and with a greater rigor in their methodology, in order to evaluate the real educational benefit of their participation and their perceptions. It is important that the scientific community generate a guide to consolidate the criteria that should be considered when selecting students as study subjects. This would undoubtedly, allow researchers, ethical review institutions and editors of scientific journals to better evaluate studies in these populations.

KEY WORDS: Students; Subject of investigation; Research ethics; Vulnerable populations.

\section{REFERENCIAS BIBLIOGRÁFICAS}

Ahumada, C. M. La libertad de investigación científica. Panorama de su situación en el constitucionalismo comparado y en el derecho internacional. Rev. Chil. Derecho, 39(2):411-45, 2012.

Aycock, M. D. \& Currie, E. R. Minimizing risks for nursing students recruited for health and educational research. Nurse Educ., 38(2):5660, 2013.

Boileau, E.; Patenaude, J. \& St-Onge, C. Twelve tips to avoid ethical pitfalls when recruiting students as subjects in medical education research. Med. Teach., 40(1):20-5, 2017.

Bradbury-Jones, C. \& Alcock, J. Nursing students as research participants: a framework for ethical practice. Nurse Educ. Today, 30(2):192-6, 2010.

Christakis, N. Do medical student research subjects need special protection? IRB, 7(3):1-4, 1985.

Clark, E. \& McCann, T. V. Researching students: an ethical dilemma. Nurse Res., 12(3):42-51, 2005.

Congreso Nacional. Ley 19.628. Sobre Protección de la Vida Privada. Santiago de Chile, Diario Oficial, Biblioteca del Congreso Nacional de Chile, Gobierno de Chile, 1999. Disponible en: https:// www.leychile.cl/Navegar?idNorma $=141599$

Congreso Nacional. Ley 20.120. Sobre la Investigación Científica en el Ser Humano, su Genoma y Prohibe la Clonación Humana. Santiago de Chile, Diario Oficial, Biblioteca del Congreso Nacional de Chile, Gobierno de Chile, 2006. Disponible en: https://www.leychile.cl/ Navegar?idNorma $=253478$

Congreso Nacional. Ley 20.584. Regula los Derechos y Deberes que tiene las Personas en Relación con Acciones Vinculadas a su Atención en Salud. Santiago de Chile, Diario Oficial, Biblioteca del Congreso Nacional de Chile, Gobierno de Chile, 2012. Disponible en: https:// www.leychile.cl/Navegar?idNorma $=1039348$

Dalziel, J. R. Students as research subjects: ethical and educational issues. Aust. Psychol., 31(2):119-23, 1996.

Dhai, A. The research ethics evolution: from Nuremberg to Helsinki. $S$. Afr. Med. J., 104(3):178-80, 2014.

Diamond, M. R. \& Reidpath, D. D. Psychology ethics down under: a survey of student subject pools in Australia. Ethics Behav., 2(2):101$8,1992$. 
Eliott, L. J.; Rice, S.; Trafimow, D.; Madson, L. \& Hipshur, M. F. Research participation versus classroom lecture: a comparison of student learning. Teach. Psychol., 37(2):129-31, 2010

Ferguson, L. M.; Myrick, F. \& Yonge, O. Ethically involving students in faculty research. Nurse Educ. Today, 26(8):705-11, 2006.

Forester, J. P. \& McWhorter, D. L. Medical students' perceptions of medical education research and their roles as participants. Acad. Med., 80(8):780-5, 2005.

Henry, R. C. \& Wright, D. E. When do medical students become human subjects of research? The case of program evaluation. Acad. Med., 76(9):871-5, 2001.

Leentjens, A. F. \& Levenson, J. L. Ethical issues concerning the recruitment of university students as research subjects. J. Psychosom. Res., 75(4):394-8, 2013a.

Leentjens, A. F. \& Levenson, J. L. Ethical issues concerning the recruitment of university students as research subjects: reply to Lumley and Jasinski. J. Psychosom. Res., 75(4):400, 2013b.

Miracle, V. A. The Belmont report: the triple crown of research ethics. Dimens. Crit. Care Nurs., 35(4):223-8, 2016.

Moher, D.; Liberati, A.; Tetzlaff, J.; Altman, D. G. \& PRISMA Group. Preferred reporting items for systematic reviews and meta-analyses: the PRISMA statement. PLoS Med., 6(7):e1000097, 2009.

Nimmer, J. G. \& Handelsman, M. M. Effects of subject pool policy on student attitudes toward psychology and psychological research. Teach. Psychol., 19(3):141-4, 1992.

Nuremberg Military Tribunal. The Nuremberg Code. JAMA, 276(20):1691, 1996.

Office for Human Research Protections, U.S. Department of Health and Human Services. International Compilation of Human Research Standards. Washington D. C., US Department of Health and Human Services, 2018. Disponible en: https://www.hhs.gov/ohrp/sites/ default/files/2018-International-Compilation-of-Human-ResearchStandards.pdf

Oficina del Alto Comisionado para los Derechos Humanos. Declaración Universal de los Derechos Humanos. Adoptada y proclamada por la Asamblea General en su resolución 217 A (III), de 10 de diciembre de 1948. Ginebra, Naciones Unidas, Oficina del Alto Comisionado para los Derechos Humanos, 2018. Disponible en: http://www.ohchr.org/EN/UDHR/Documents/UDHR_Translations/ spn.pdf

Organización Panamericana de la Salud \& Consejo de Organizaciones Internacionales de las Ciencias Médicas. Pautas Éticas Internacionales para la Investigación relacionada con la Salud con Seres Humanos. $4^{a}$ ed. Ginebra, Consejo de Organizaciones Internacionales de las Ciencias Médicas (CIOMS), 2016. Disponible en: https:// cioms.ch/w p-content/uploads/2017/12/CIOMSEthicalGuideline_SP_INTERIOR-FINAL.pdf

Rice, T. W. The historical, ethical, and legal background of humansubjects research. Respir. Care, 53(10):1325-9, 2008.

Ridley, R. T. Assuring ethical treatment of students as research participants. J. Nurs. Educ., 48(10):537-41, 2009.

Roberts, L. D. \& Allen, P. J. A brief measure of student perceptions of the educational value of research participation. Aust. J. Psychol., 65(1):22-9, 2013

Sarpel, U.; Hopkins, M. A.; More, F.; Yavner, S.; Pusic, M.; Nick, M. W.; Song, H.; Ellaway, R. \& Kalet, A. L. Medical students as human subjects in educational research. Med. Educ. Online, 18:19524, 2013.

Scheppler, J. \& Kolar, C. Your IRB: Educating students, monitoring student research, and safeguarding students as research subjects. NCSSSMST. J., 13(2):19-29, 2008.

Shannon, T. A. Should medical students be research subjects? IRB, 1(2):4, 1979.

Sullivan, G. M. Education research and human subject protection: crossing the IRB Quagmire. J. Grad. Med. Educ., 3(1):1-4, 2011.

Sykes, L. M. \& Dullabh, H. Students' vulnerability in educational research. S. A. D. J., 67(5):222, 224-7, 2012.
Vaidya, P.; Kamat, S.; Shetty, Y. \& Singh, K. N. Is Coercion involved in the decision-making of medical students participating in research?: A cross-sectional study. Asian Bioeth. Rev., 8(1):20-36, 2016.

Valenzuela, S.; Aliaga, V.; Burdiles, P.; Carvallo, A.; Díaz, E.; Guerrero, M.; Rueda, L. \& Valenzuela, C. Reflexiones en torno a la ley $\mathrm{N}^{\circ} 20.584$ y sus implicancias para la investigación biomédica en Chile. Rev. Med. Chile, 143(1):96-100, 2015.

van Delden, J. J. \& van der Graaf, R. Revised CIOMS international ethical guidelines for health-related research involving humans. JAMA, 317(2):135-6, 2017

Voo, T. C. Using medical students as research subjects: is it ethical? Ann. Acad. Med. Singapore, 38(12):1019-20, 2009.

Walsh, K. Medical students as human subjects in educational research - the importance of responder bias. Med. Educ. Online, 18(1):20757, 2013.

World Medical Association. World Medical Association Declaration of Helsinki Ethical Principles for Medical Research Involving Human Subjects. JAMA, 310(20):2191-4, 2013.

Dirección para correspondencia:

Prof. Dr. Ramón Fuentes Fernández

Centro de Investigación en Ciencias Odontológicas

Facultad de Odontología

Universidad de La Frontera

Av. Francisco Salazar 1145

Temuco

CHILE

E-mail: ramon.fuentes@ufrontera.cl

Recibido : 08-05-2018

Aceptado: 12-09-2018 